DEMOGRAPHIC RESEARCH

VOLUME 33, ARTICLE 20, PAGES 561-588

PUBLISHED 16 SEPTEMBER 2015

http://www.demographic-research.org/Volumes/Vol33/20/

DOI: $\quad$ 10.4054/DemRes.2015.33.20

Research Article

Lifetime reproduction and the second

demographic transition: Stochasticity and individual variation

Silke van Daalen

Hal Caswell

(C) 2015 Silke van Daalen \& Hal Caswell.

This open-access work is published under the terms of the Creative Commons Attribution NonCommercial License 2.0 Germany, which permits use, reproduction \& distribution in any medium for non-commercial purposes, provided the original author(s) and source are given credit. See http://creativecommons.org/licenses/by-nc/2.0/de/ 


\section{Table of Contents}

$1 \quad$ Introduction $\quad 562$

1.1 Individual stochasticity and the sources of variance 563

$2 \quad$ Methodology $\quad 565$

$2.1 \quad$ Markov chains with rewards $\quad 565$

2.1.1 Lifetime accumulated rewards $\quad 566$

\begin{tabular}{ll}
2.2 & Data: fertility and mortality \\
\hline
\end{tabular}

$\begin{array}{ll}2.3 & \text { Characterizing patterns of LRO }\end{array}$

3 Results $\quad 570$

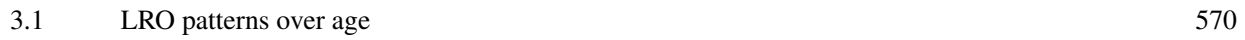

$\begin{array}{ll}3.2 & \text { Patterns over time } \\ 3.3 & 571\end{array}$

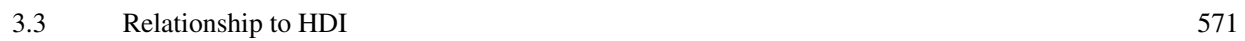

$\begin{array}{ll}3.4 & \text { Relationships among the statistics of LRO }\end{array}$

$4 \quad$ Discussion $\quad 576$

$\begin{array}{ll}4.1 & \text { Individual stochasticity and its components }\end{array}$

$\begin{array}{ll}4.2 & \text { Individual stochasticity }\end{array}$

$5 \quad$ Acknowledgements $\quad 582$

$\begin{array}{ll}\text { References } & 583\end{array}$

$\begin{array}{lll}\text { A } & \text { Appendix } & 587\end{array}$ 


\title{
Lifetime reproduction and the second demographic transition: Stochasticity and individual variation
}

\author{
Silke van Daalen ${ }^{1}$ \\ Hal Caswell ${ }^{2}$
}

\begin{abstract}
BACKGROUND

In the last half of the previous century many developed countries went through a period of decreasing fertility rates, referred to as the second demographic transition. This transition is often measured using the Total Fertility Rate (TFR), which gives the mean number of children produced by a woman surviving through her reproductive years. The TFR ignores effects of mortality and, as a mean, provides no information on variability among individuals in lifetime reproduction.
\end{abstract}

\section{OBJECTIVE}

Our goal is to quantify the statistics (mean, variance, standard deviation, coefficient of variation, and skewness) during the second demographic transition. We compare these statistical properties as functions of age, time, and developmental indices.

\section{METHODS}

We used Markov chains with rewards to compute the moments of lifetime reproductive output (LRO) based on age-specific mortality and fertility rates for 40 developed countries, two hunter-gatherer populations and a group of North-American Hutterites. The analysis uses a Markov chain to model individual survival, and treats reproduction as a Bernoulli-distributed reward with probability equal to the age-specific fertility.

\section{RESULTS}

All statistical properties of lifetime reproduction changed during the transition. The mean and standard deviation of LRO declined, and the coefficient of variation and skewness increased. By 2000, these statistics were tightly correlated across countries, suggesting that the entire distribution of LRO shifted, not just the mean.

\footnotetext{
${ }^{1}$ Institute for Biodiversity and Ecosystem Dynamics (IBED), University of Amsterdam, Science Park 904, 1098 XH Amsterdam, The Netherlands, E-Mail: silkevandaalen@hotmail.com.

${ }^{2}$ Institute for Biodiversity and Ecosystem Dynamics (IBED), University of Amsterdam, Science Park 904, 1098 XH Amsterdam, The Netherlands, E-Mail: H.Caswell@uva.nl.
} 
van Daalen \& Caswell: Lifetime reproduction and the second demographic transition

\section{CONCLUSIONS}

We find that developed countries adhere to a seemingly universal distribution in LRO, during and after the second demographic transition. This distribution becomes more apparent when development improves health circumstances and decreases mortality.

\section{Introduction}

During the twentieth century, many countries experienced the so-called second demographic transition, showing sharp declines in the population's fertility level. The demographic transition is characterized both by a decline in the number of children and a postponement in age of first childbearing, with declining mortality rates being a main driver and component of the transition (Liu, Rotkirch, and Lummaa 2012). Lower fertility levels are the main feature of the first demographic transition, which occurred in most European countries, starting in the latter half of the 19th century. The second demographic transition, starting around 1970 and characterized by increased postponement of first reproduction, saw fertility decline even more sharply, resulting in an increasing number of countries dropping below replacement level (2.1 children per female) (Lee 2003). In 2003, more than $50 \%$ of the world's population lived in countries with below replacement fertility (Wilson 2004).

Countries in Southern and Eastern Europe and in East Asia have reached even lower levels of fertility, dropping below 1.3 (i.e. "lowest-low fertility") (Goldstein, Sobotka, and Jasilioniene 2009; Wilson 2004). In recent years, fertility has started to increase again. Myrskylä, Kohler, and Billari (2009) show that, although the relationship between Total Fertility Rate and the Human Development Index was negative in the past, this relationship has become positive in highly developed countries, resulting in increasing fertility.

Explanations for these fertility declines during the transition include the effects of improving socioeconomic circumstances, tempo effects related to postponement of childbearing, better access to methods of fertility control, and diffusion of ideas about family planning at the population level (Hill and Kaplan 1999; Kirk 1996; Bryant 2007; Goldstein, Sobotka, and Jasilioniene 2009). Biodemographic explanations have been proposed that explain reduced fertility as a (perhaps mistaken) evolved response to increased costs of offspring (Hill and Kaplan 1999). Recent reports of recovering fertility provide similar explanations for rising fertility levels; the effect of even further improvement in socioeconomic circumstances, decreased tempo effects and perhaps, in some cases, effect of government policies to raise national fertility (Goldstein, Sobotka, and Jasilioniene 2009; Myrskylä, Kohler, and Billari 2009).

In this paper we are interested in lifetime reproductive output (LRO): the number of 
offspring produced by a woman over her entire life. Remarkably, there is no established term for this quantity in human demography. ${ }^{3}$ Lifetime reproductive output is, however, related to some familiar demographic quantities. The net reproductive rate $R_{0}$ is the expectation of LRO (usually restricted to female offspring). The net reproductive rate is also the population growth rate per generation, and indicates whether a population can persist, grow, or decline (Lotka 1936; Woofter 1949; Cushing and Zhou 1994; Caswell 2009). The total fertility rate TFR is the expectation of LRO, conditional on survival to the end of childbearing age (Le Bras 2008). Lifetime reproductive output is the quantity of which $R_{0}$ and TFR are conditional expectations.

We refer to lifetime reproductive output rather than births because reproductive output is not always equivalent to births. In anthropological demography, reproduction is often quantified not in terms of births, but in terms of the production of offspring who survive to some age (e.g., to age 15 in Hill and Hurtado (1996)). We are also interested in lifetime reproductive output in species other than humans, where the hatching of eggs, the germinating of seeds, etc. are not well-described by the term "births." Our methods are easily applicable to such cases.

Our goal is to calculate not only the expectation, but also the inter-individual variation in LRO. That variation is implied by, and can be calculated from, a set of demographic rates. Because they are expectations, neither $R_{0}$ nor the TFR provide any information on variation among individuals, and it is presently unknown how inter-individual variation in fertility changed during the second demographic transition and in response to changes in socioeconomic conditions (e.g. Myrskylä, Kohler, and Billari (2009)).

Inter-individual variation in LRO can be quantified by several statistics. The variance and standard deviation measure variation on an absolute scale. The coefficient of variation $(\mathrm{CV})$ scales the standard deviation relative to the mean. The standardized variance, also known as Crow's $I$, scales the variance relative to the square of the mean (Crow 1958). Crow's $I$ measures the opportunity for selection on a varying trait and provides an upper limit to the strength of selection. Skewness in LRO measures the asymmetry of the distribution. If skewness is positive, as is often the case with fertility in animal studies (Clutton-Brock 1988), many individuals produce few children, and a long tail of rare individuals producing many children.

\subsection{Individual stochasticity and the sources of variance}

Inter-individual variation in LRO may arise from individual stochasticity or individual heterogeneity. Individual stochasticity (Caswell 2009) is the random variation among individuals in the outcomes of applying identical vital rates. Individual stochasticity is inherent in any set of mortality and fertility rates, and given those rates, we can calculate

\footnotetext{
${ }^{3}$ Ecologists and evolutionary biologists sometimes also use the term "lifetime reproductive success."
} 
van Daalen \& Caswell: Lifetime reproduction and the second demographic transition

the consequences of these stochastic events (Caswell 2009, 2011, 2014a; Caswell and Kluge 2015). Individual stochasticity has been found to be a major contributor to variance in LRO in many species (Caswell 2011; Tuljapurkar, Steiner, and Orzack 2009; Steiner and Tuljapurkar 2012).

Heterogeneity, in contrast, refers to differences in the characteristics of individuals, and hence in their vital rates, within the same age or stage. To calculate the consequences of this heterogeneity requires a model that incorporates the heterogeneity, as frailty models do in the study of mortality (e.g., Vaupel, Manton, and Stallard 1979; Caswell 2014a). The calculated variance due to individual stochasticity can serve as a null model for comparison with observed measures of variance (Tuljapurkar, Steiner, and Orzack 2009; Steiner and Tuljapurkar 2012).

Individual stochasticity contributes to inter-individual variation in LRO in two ways. First, individuals will differ in the pathways they follow throughout the life cycle; by chance some will live longer while some die sooner. Second, individuals of a given age will experience stochasticity in their reproductive output; given a probability of reproduction, by chance some will produce a child and some will not. The overall variance in LRO can be partitioned into contributions from these two sources, as we will see in Section 4.

Caswell (2011) presented a method to calculate the mean, variance and other statistical properties of LRO due to the individual stochasticity implied by a mortality and fertility schedule. The method uses a Markov chain description of the life cycle, assigns a random reward (in our case, reproduction) to each transition, and then accumulates this reward over the life cycle (Howard 1960; Caswell 2011).

In this paper, we will assess changes in the statistics of LRO during the second demographic transition, based on mortality and fertility data from 40 developed countries, covering the years 1891 to 2011, but with a focus on the period between 1970 and 2012. As is customary in studies of this transition, we rely on period fertility and mortality data (e.g., Goldstein, Sobotka, and Jasilioniene 2009; Bongaarts and Sobotka 2012). We compute the mean, variance, standard deviation, coefficient of variation $(\mathrm{CV})$, and skewness of LRO. These are assessed over age, over time, in relation to human development, and in relation to the other statistical properties. We will compare the statistics of LRO for our sample of developed countries with those for several populations without fertility control. The latter include the hunter-gatherer populations of the Ache and the Hadza, and the high-fertility population of the Hutterites.

Over time, we assess the changes in all statistics during the second demographic transition, focusing on the period between 1960 and 2011, which saw the steepest declines, lowest levels of fertility, and the start of a possible fertility recovery (Goldstein, Sobotka, and Jasilioniene 2009; Myrskylä, Kohler, and Billari 2009). Following Myrskylä, Kohler, and Billari (2009), we also investigate the relationship between the statistics of LRO and the Human Development Index (HDI) of the United Nations Development Programme. However, where Myrskylä, Kohler, and Billari (2009) focus on the the effect of 
an increasing HDI on TFR, we assess the effect of HDI on multiple statistics of lifetime reproduction.

\section{Methodology}

Notation. Matrices are denoted by upper-case bold symbols (e.g., P), vectors by lowercase bold symbols (e.g., $\boldsymbol{\rho}$ ). Vectors are column vectors by default. The transpose of $\mathbf{P}$ is $\mathbf{P}^{\top}$. The inverse of $\mathbf{P}$ is $\mathbf{P}^{-1}$. The vector $\mathbf{1}$ is a vector of ones, and the matrix $\mathbf{I}$ is the identity matrix. Where necessary to avoid confusion, dimensions are indicated by subscripts; e.g., the $\omega \times \omega$ identity matrix is $\mathbf{I}_{\omega}$. The diagonal matrix with the vector $\mathbf{x}$ on the diagonal and zeros elsewhere is denoted $\mathcal{D}(\mathbf{x})$. The expected value is denoted by $E(\cdot)$. The Hadamard, or element-by-element, product of matrices $\mathbf{A}$ and $\mathbf{B}$ is denoted by $\mathbf{A} \circ \mathbf{B}$. Transition matrices of Markov chains are written in column-to-row orientation, and hence their columns sum to one.

\subsection{Markov chains with rewards}

Our analysis describes the life cycle as an absorbing Markov chain (e.g., Caswell 2001, 2006, 2009; see Feichtinger 1973 for an early example). It is applicable to age-structured and stage-structured models and to models incorporating various types of temporal or environmental variation. In our case, age-structured population projection matrices are transformed into a Markov chain to represent the human life cycle. Let $\omega$ denote the number of age classes. Death is incorporated into the model as an absorbing state. The Markov chain transition matrix is

$$
\mathbf{P}=\left(\begin{array}{c|c}
\mathbf{U} & 0 \\
\hline \mathbf{m}^{\top} & 1
\end{array}\right)
$$

where $\mathbf{U}$ is a $\omega \times \omega$ matrix of transition probabilities among transient (i.e., living) states, and $\mathbf{m}^{\top}$ is a $1 \times \omega$ vector of mortality rates. The matrix $\mathbf{U}$ contains survival probabilities on the subdiagonal and zeros elsewhere; e.g., for $\omega=3$,

$$
\mathbf{U}=\left(\begin{array}{ccc}
0 & 0 & 0 \\
P_{1} & 0 & 0 \\
0 & P_{2} & 0
\end{array}\right)
$$

Reproduction appears as a "reward" associated with the transitions between the states of the Markov chain. Individuals moving from age $j$ to age $i$ collect the reward $r_{i j}$ (Howard 1960; Caswell 2011). In demography (e.g., in population projections and the Euler-Lotka equation) age-specific fertility depends only on the current age; $r_{i j}$ depends 
van Daalen \& Caswell: Lifetime reproduction and the second demographic transition

on $j$ but not on the transition made between $j$ and $i{ }^{4}$ We consider $r_{i j}$ to be a random variable with a Bernoulli distribution (Caswell 2011), thus ignoring multiple births:

$$
r_{i j}=\left\{\begin{array}{l}
1 \text { with probability } f_{j} \\
0 \text { with probability }\left(1-f_{j}\right)
\end{array}\right.
$$

where the probabilities $f_{j}$ are age-specific fertilities. We assume that individuals in the absorbing state accrue no rewards (i.e., the dead do not reproduce).

Calculating the statistical properties of lifetime reproductive output requires a set of matrices giving the moments of the reward for each transition; we call these reward matrices. That is, $\mathbf{R}_{k}$ is a matrix of the $k$ th moments of the transition-specific rewards $r_{i j}$. The first moment matrix is

$$
\mathbf{R}_{1}=\left(\begin{array}{ccc|c}
f_{1} & \ldots & f_{\omega} & 0 \\
\vdots & \ddots & \vdots & \vdots \\
f_{1} & \ldots & f_{\omega} & 0 \\
\hline f_{1} & \ldots & f_{\omega} & 0
\end{array}\right)
$$

where the upper right block is of dimension $\omega \times \omega$. Under the Bernoulli assumption, the higher-order moments are equal:

$$
\mathbf{R}_{1}=\mathbf{R}_{2}=\mathbf{R}_{3}
$$

\subsubsection{Lifetime accumulated rewards}

We define $\rho$ as a vector, of dimension $(\omega+1) \times 1$, of accumulated rewards for each initial age. The entries in the first age class (age 0 ) refer to accumulated reproduction over the entire lifetime of the individual. The $i$ th entry of $\rho$ describes the accumulation over the remaining lifetime of an individual of age $i$. The vector of $k$ th moments of $\rho$ is denoted $\rho_{k}$, where

$$
\boldsymbol{\rho}_{k}=\left(E\left[\rho_{i}^{k}\right]\right)
$$

From the recursion equations presented in Caswell (2011), we obtain equations for the equilibria of $\rho_{k}$ (Caswell and van Daalen 2015, in prep.) Because the absorbing state accumulates no rewards, we are interested only in the subvector $\tilde{\boldsymbol{\rho}}$ giving the accumulation of rewards in the $\omega$ transient states. To this end, we define a matrix $\mathbf{Z}$

$$
\mathbf{Z}=\left(\mathbf{I}_{\omega} \mid \mathbf{0}_{\omega \times 1}\right)
$$

\footnotetext{
${ }^{4}$ See Caswell (2014b) for a multistate model in which reproduction depends on age and parity, and rewards are explicitly associated with transitions among parity states.
} 
Multiplying $\boldsymbol{\rho}_{i}$ by $\mathbf{Z}$ cleaves off the rewards for the absorbing states, leaving only the rewards for the transient states of the Markov chain. The equilibria for the first three moments of accumulated rewards are as follows:

$$
\begin{aligned}
\tilde{\boldsymbol{\rho}}_{1} & =\mathbf{N}^{\top} \mathbf{Z}\left(\mathbf{P} \circ \mathbf{R}_{1}\right)^{\top} 1_{\omega+1} \\
\tilde{\boldsymbol{\rho}}_{2} & =\mathbf{N}^{\top}\left[\mathbf{Z}\left(\mathbf{P} \circ \mathbf{R}_{2}\right)^{\top} 1_{\omega+1}+2\left(\mathbf{U} \circ \mathbf{R}_{1}\right)^{\top} \tilde{\boldsymbol{\rho}}_{1}\right] \\
\tilde{\boldsymbol{\rho}}_{3} & =\mathbf{N}^{\top}\left[\mathbf{Z}\left(\mathbf{P} \circ \mathbf{R}_{3}\right)^{\top} 1_{\omega+1}+3\left(\mathbf{U} \circ \mathbf{R}_{2}\right)^{\top} \tilde{\boldsymbol{\rho}}_{1}+3\left(\mathbf{U} \circ \mathbf{R}_{1}\right)^{\top} \tilde{\boldsymbol{\rho}}_{2}\right]
\end{aligned}
$$

where $\mathbf{N}=\left(\mathbf{I}_{\omega}-\mathbf{U}\right)^{-1}$ is the fundamental matrix of the Markov chain. The entries of the first moment vector $\tilde{\boldsymbol{\rho}}_{1}$ give the mean remaining lifetime reproductive output of each age class. The other statistical properties of variance, standard deviation, coefficient of variation, and skewness of lifetime reproductive output are calculated from the moment vectors in the following way:

$$
\begin{aligned}
V(\tilde{\boldsymbol{\rho}}) & =\tilde{\boldsymbol{\rho}}_{2}-\tilde{\boldsymbol{\rho}}_{1} \circ \tilde{\boldsymbol{\rho}}_{1} \\
S D(\tilde{\boldsymbol{\rho}}) & =\sqrt{V(\tilde{\boldsymbol{\rho}})} \\
C V(\tilde{\boldsymbol{\rho}}) & =\mathcal{D}\left(\tilde{\boldsymbol{\rho}}_{1}\right)^{-1} S D(\tilde{\boldsymbol{\rho}}) \\
S k(\tilde{\boldsymbol{\rho}}) & =\mathcal{D}[V(\tilde{\boldsymbol{\rho}})]^{-3 / 2}\left(\tilde{\boldsymbol{\rho}}_{3}-3 \tilde{\boldsymbol{\rho}}_{1} \circ \tilde{\boldsymbol{\rho}}_{2}+2 \tilde{\boldsymbol{\rho}}_{1} \circ \tilde{\boldsymbol{\rho}}_{1} \circ \tilde{\boldsymbol{\rho}}_{1}\right) .
\end{aligned}
$$

\subsection{Data: fertility and mortality}

We obtained data on period survival and fertility from the Human Mortality Database (Human Mortality Database 2014), the Human Fertility Database (Human Fertility Database 2014) and the Human Fertility Collection (Human Fertility Collection 2014). These agespecific data were available for 40 developed countries for varying numbers of years (Table 1).

For comparison with these developed countries, we analyzed two hunter-gatherer populations: the Hadza of Tanzania and the Ache of Paraguay, using mortality and fertility data from Gurven and Kaplan (2007), Blurton Jones (2011), and Hill and Hurtado (1996). The Hadza live in the sub-Saharan wooded savanna near Serengeti National Park. Women reproduce after marriage, starting at age 14 and peaking in their reproductive output around age 30 (Blurton Jones 2011). The Ache live in the subtropical Paraná watershed of Eastern Paraguay. Ache women start reproducing at age 12 and reach a peak in reproduction around age 30-35 (Hill and Hurtado 1996). Both Ache and Hadza populations are exposed to higher mortality than countries in the developed world, resulting in life expectancies of 37 and 34 years, respectively (Gurven and Kaplan 2007). We also 
van Daalen \& Caswell: Lifetime reproduction and the second demographic transition

analyzed the ethnic Hutterites of North America, an Anabaptist religious sect with unregulated fertility reported to have the highest TFR of any known population (Eaton and Mayer 1953). We used Hutterite fertility rates from a study by Eaton and Mayer (1953) covering the period of 1946-1950. We follow Eaton and Mayer in assuming that Hutterite mortality was similar to the overall U.S. rates during this period.

\subsection{Characterizing patterns of LRO}

The computation of LRO statistics from the available data permits many different comparisons. We will consider LRO by age, LRO over time, LRO in relation to socio-economic indicators, and the relationship among the different statistics in LRO. Here, we provide more detail of what each of these comparisons entails.

We will present the mean, standard deviation (SD), coefficient of variation (CV), and skewness (Sk) of remaining LRO as a function of age, for a fixed year. Over time, we will show the patterns in mean LRO, standard deviation, coefficient of variation and skewness in LRO at birth for each country. Our focus lies on the period from 1965 to 2010, the period characteristically associated with the second demographic transition. We assess whether our results for LRO are similar to known results using TFR and whether similar patterns arise in the other statistics of LRO.

The relationship between LRO and socio-economic indicators is investigated using the Human Development Index, as LRO presumably responds to the conditions in which individuals find themselves. The HDI, as employed by the United Nations Development Programme, measures a country's health, education and standard of living. These measures are assigned equal weight and combined into a broad-scale indicator of human development (United Nations Development Programme 2014). Myrskylä, Kohler, and Billari (2009) found a relationship between period TFR and the human development index (HDI). Increases in the HDI up to $\sim 0.9$ were associated with declines in TFR, but above that point, they found evidence that the TFR began to increase. To evaluate such changes for the mean and variation, we regressed the statistics of LRO for all countries, at age 0, against the HDI for the years 1980 and 2009.

When viewed across countries or over time, the statistics of LRO show clear and non-random relationships among themselves. We examine these by looking for correlations among the statistics and examining temporal trajectories in the statistics. 
Table 1: $\quad$ Countries used in the analyses

\begin{tabular}{rlrrl}
\hline$\#$ & Country & Data Range & Years & Sources \\
& & & & \\
\hline 1 & Australia & $1921-2009$ & 89 & HMD, HFC \\
2 & Austria & $1951-2010$ & 60 & HMD, HFD \\
3 & Belarus & $1960-2008$ & 47 & HMD, HFC \\
4 & Belgium & $1952-2009$ & 58 & HMD, HFC \\
5 & Bulgaria & $1947-2009$ & 63 & HMD, HFD \\
6 & Canada & $1921-2009$ & 89 & HMD, HFD \\
7 & Czech Republic & $1950-2011$ & 62 & HMD, HFD \\
8 & Denmark & $1901-2011$ & 111 & HMD, HFC \\
9 & East Germany & $1956-2010$ & 55 & HMD, HFD \\
10 & England and Wales $1938-2009$ & 72 & HMD, HFD \\
11 & Estonia & $1959-2010$ & 52 & HMD, HFD \\
12 & Finland & $1939-2009$ & 71 & HMD, HFD \\
13 & France & $1946-2010$ & 65 & HMD, HFD \\
14 & Germany & $1990-2010$ & 21 & HMD, HFD \\
15 & Hungary & $1950-2009$ & 60 & HMD, HFD \\
16 & Iceland & $1963-2009$ & 47 & HMD, HFC \\
17 & Ireland & $1955-2009$ & 55 & HMD, HFC \\
18 & Italy & $1930-2009$ & 80 & HMD, HFC \\
19 & Japan & $1947-2009$ & 63 & HMD, HFD \\
20 & Latvia & $1970-2011$ & 42 & HMD, HFC \\
21 & Lithuania & $1959-2010$ & 52 & HMD, HFD \\
22 & Luxembourg & $1966-2009$ & 44 & HMD, HFC \\
23 & Netherlands & $1950-2009$ & 60 & HMD, HFD \\
24 & New Zealand & $1948-2008$ & 61 & HMD, HFC \\
25 & Northern Ireland & $1974-2009$ & 36 & HMD, HFD \\
26 & Norway & $1967-2009$ & 43 & HMD, HFD \\
27 & Poland & $1970-2009$ & 40 & HMD, HFC \\
28 & Portugal & $1940-2009$ & 70 & HMD, HFD \\
29 & Russia & $1959-2010$ & 52 & HMD, HFD \\
30 & Scotland & $1938-2009$ & 65 & HMD, HFD \\
31 & Slovakia & $1950-2009$ & 60 & HMD, HFD \\
32 & Slovenia & $1983-2009$ & 27 & HMD, HFD \\
33 & Spain & $1922-2009$ & 88 & HMD, HFC \\
34 & Sweden & $1891-2010$ & 120 & HMD, HFD \\
35 & Switzerland & $1932-2011$ & 80 & HMD, HFD \\
36 & Taiwan & $1976-2010$ & 35 & HMD, HFD \\
37 & Ukraine & $1959-2009$ & 51 & HMD, HFD \\
38 & United Kingdom & $1974-2009$ & 36 & HMD, HFD \\
39 & USA & $1933-2010$ & 78 & HMD, HFD \\
40 & West Germany & $1956-2010$ & 55 & HMD, HFD \\
\hline & HMand & & & \\
\hline 3 & & & \\
\hline & & & &
\end{tabular}

Notes: Sources refer to the databases from which we collected the data; HMD for the Human Mortality Database, HFD for the Human Fertility Database and HFC for the Human Fertility Collection. 
van Daalen \& Caswell: Lifetime reproduction and the second demographic transition

\section{Results}

\subsection{LRO patterns over age}

In Figure 1, the mean and standard deviation (SD) of remaining LRO are shown as a function of age. After age 20, all populations show a decline in both mean and SD, until women reach the age of infertility around age 45-50. The Hutterites show a slight increase in mean LRO between age 0 and age 1 . In the two hunter-gatherer populations, mean remaining LRO increases with age between birth and age 20 . These increases reflect the high infant mortality rates in these populations. The SD of remaining LRO decreases almost linearly with age for the Ache and Hadza.

In Figure 2 the coefficient of variation (CV) and skewness (Sk) in remaining LRO are shown separately for developed countries and for the hunter-gatherers and Hutterites. The relative variation in remaining LRO, as measured by the CV, is between 0.5 and 1 at birth for the developed countries, but rises rapidly with age after age 25 . The remaining LRO of women over age 40 is extremely variable; by age 45 the $\mathrm{CV}$ peaks at values between 40 and a little over 300 . Hutterite lifetime $\mathrm{CV}$ is the lowest measured, falling just below 0.4. Ache lifetime CV is just below 1, whereas the Hadza are the only population with a $\mathrm{CV}$ at birth over 1 .

\section{Figure 1: $\quad$ Mean and standard deviation of LRO as a function of age}

(a) Mean

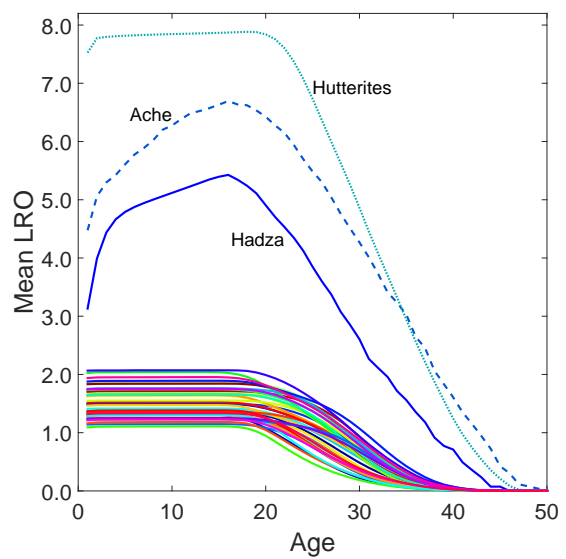

(b) Standard Deviation

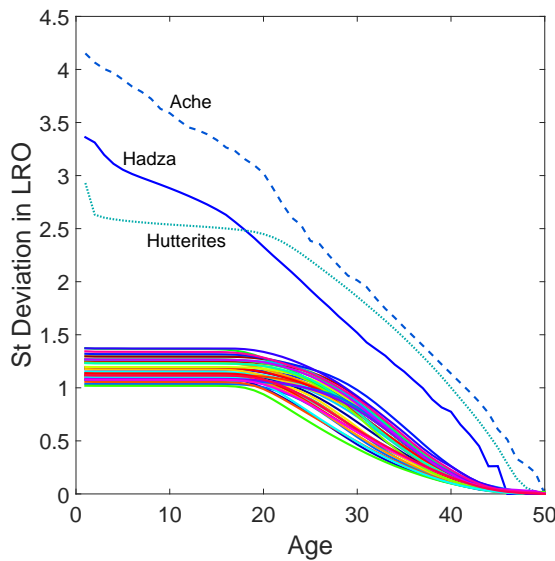

Notes: Mean and standard deviation of age-specific remaining lifetime reproductive output for 40 developed countries in the year 2000 (colored lines). The blue lines show these measures for high-fertility populations: 2 populations of hunter-gatherers, the Ache (dashed line) and the Hadza (solid line), and a population of Hutterites (dotted line). 
The skewness of remaining LRO follows a similar pattern. Skewness at birth in the developed countries is slightly positive (between 0.5 and 1 ) and increases dramatically at older ages. Skewness in LRO at birth is slightly negative for the Hutterites, and remains so until after age 20. For the Hadza and Ache, skewness starts of between 0 and 1, drops to slightly negative values, then becomes positive again around age 20. Hadza and Ache women show lower peaks in CV and skewness around age 45, whereas Hutterites show variability comparable to developed countries at this age.

In Figure 3, the lifetime values for mean, standard deviation, CV and skewness of LRO at birth are shown for all 40 countries in the year 2000, corresponding to the values in the age-dependent graphs at age 0. Mean LRO was below replacement (2.1) in 2000 for all countries.

\subsection{Patterns over time}

We focus on the period during which most developed countries experienced the second demographic transition (1965-2010). Our results for mean LRO agree with other wellknown results concerning the transition: LRO declines sharply and then begins to rise again in recent years (Goldstein, Sobotka, and Jasilioniene 2009; Myrskylä, Kohler, and Billari 2009). Measures of variability, however, display different patterns. The standard deviation of LRO also declines sharply from 1965 to about 2000, and shows signs of beginning to recover from 2000-2010. The coefficient of variation increases from 1965, levelling off after 2000 . The skewness does the same, showing a very similar pattern to the $\mathrm{CV}^{5}$

The magnitude of increase or decrease in statistical properties of LRO differs between different countries. Moreover, not all countries show a reversal in pattern in the last 5-10 years. The time series for mean and standard deviation appear similar for all countries, and also inversely similar to $\mathrm{CV}$ and skewness.

We have included a gallery showing the time series of the statistics of LRO at selected ages, for all 40 developed countries, in an Online Supplement.

\subsection{Relationship to HDI}

The HDI is a synthetic index designed to describe socioeconomic living conditons. The decline in TFR during the transition has been associated with improvement in standards of living. Myrskylä, Kohler, and Billari (2009) found that TFR declined with increases in the HDI up to a point, but that further increases in the HDI were associated with increases in TFR.

\footnotetext{
${ }^{5}$ The similarity of values of the coefficient of variation and of skewness was noted in several species by Caswell (2011). There are several possible explanations, related to possible statistical distributions of lifetime reproduction; e.g., if LRO followed a Poisson distribution, the CV would equal the skewness.
} 
van Daalen \& Caswell: Lifetime reproduction and the second demographic transition

Figure 2: $\quad$ CV and skewness of LRO as a function of age

(a) $\quad$ CV: developed countries

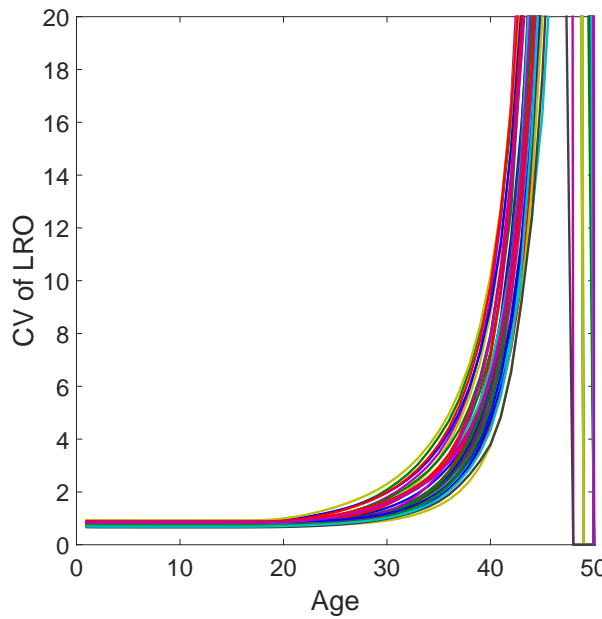

(c) Skewness: developed countries

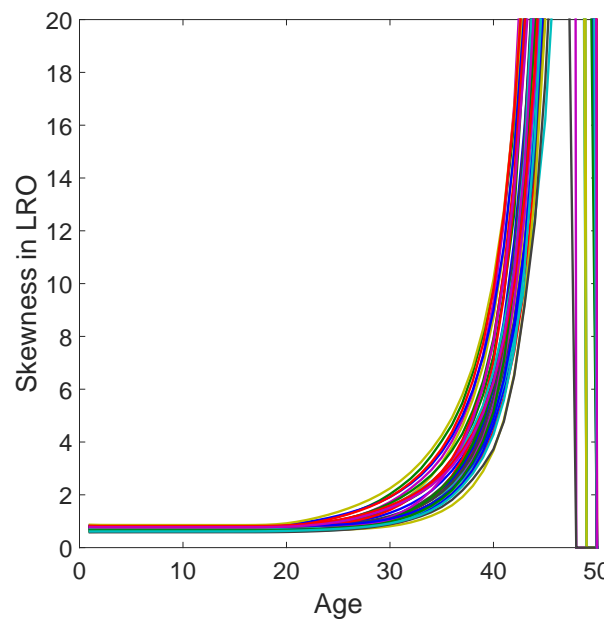

(b) $\quad \mathrm{CV}$ : high-fertility populations

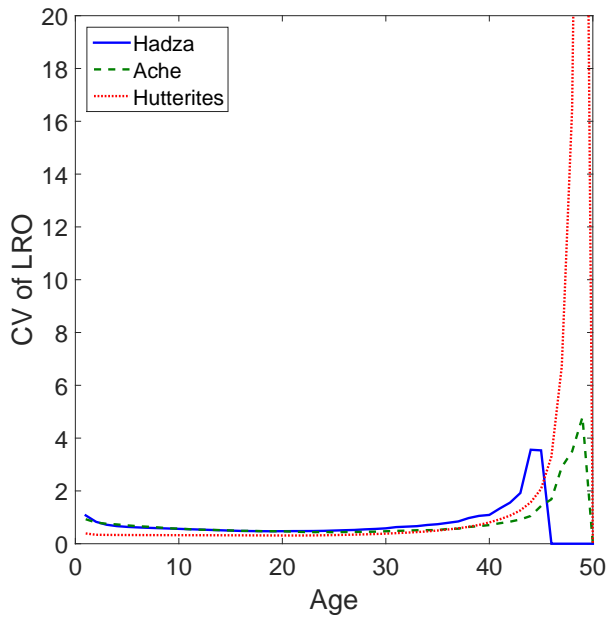

(d) Skewness: high-fertility populations

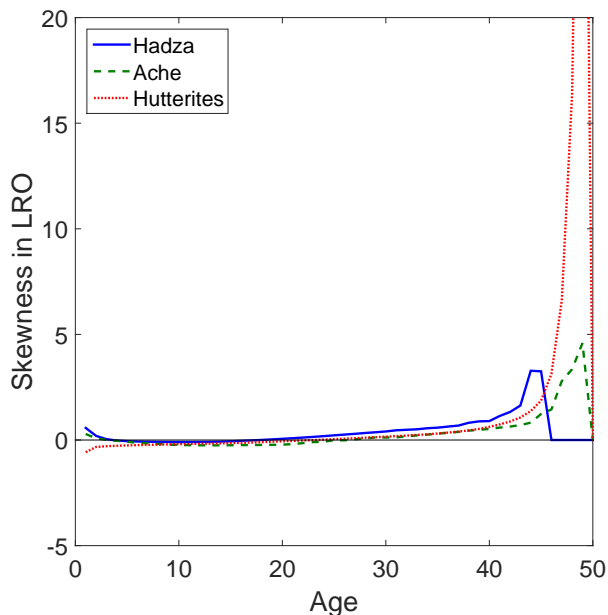

Notes: Coefficient of variation and skewness of age-specific remaining lifetime reproductive output for 40 developed countries in the year 2000 ((a),(c)), as well as for two hunter-gatherer populations, namely the Ache (dashed line) and the Hadza (solid line), and a population of Hutterites (dotted lines) ((b),(d)). 
Demographic Research: Volume 33, Article 20
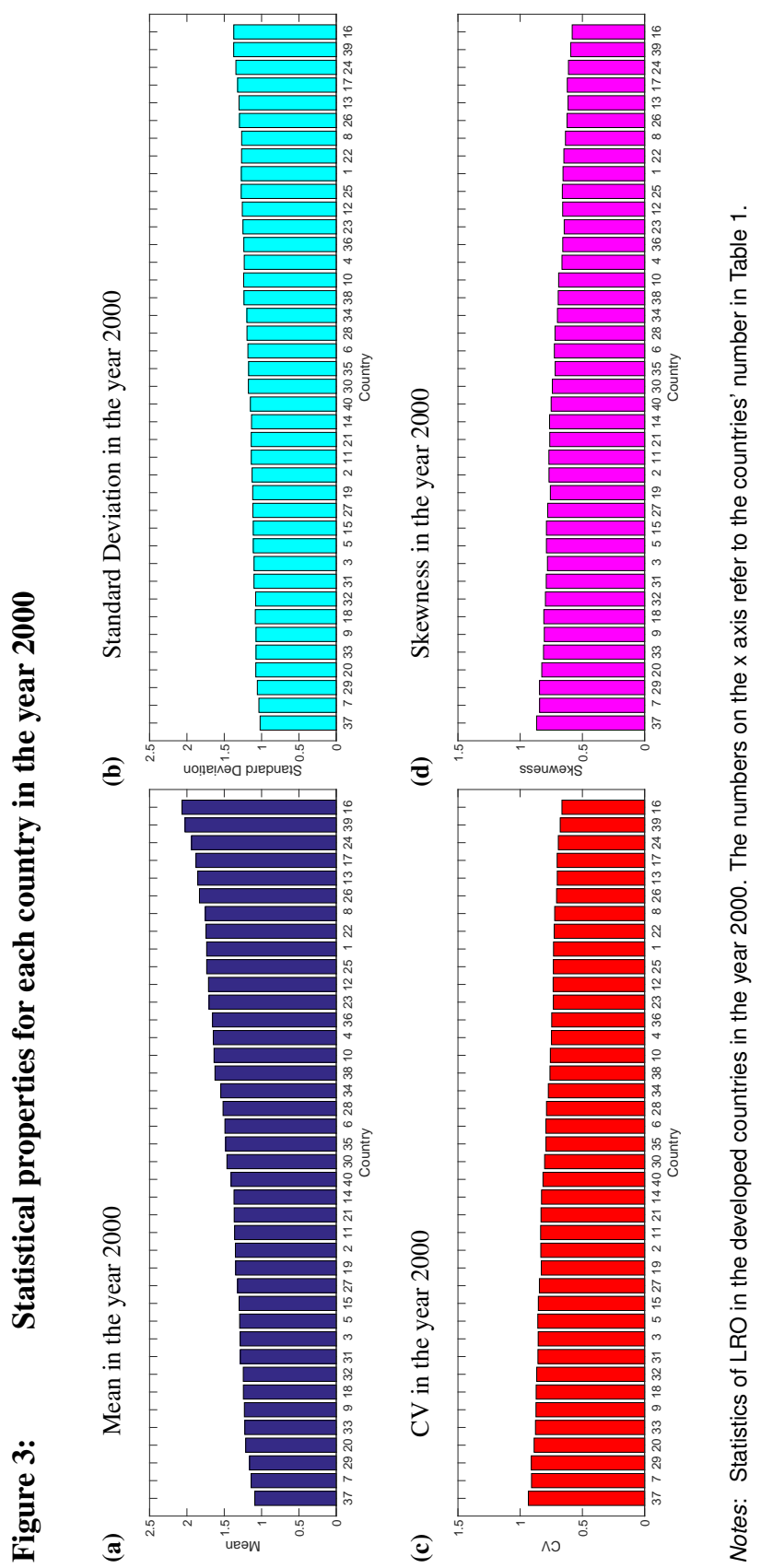

http://www.demographic-research.org 
van Daalen \& Caswell: Lifetime reproduction and the second demographic transition

\section{Figure 4: $\quad$ Statistical properties of LRO across the second demographic transition}

(a)

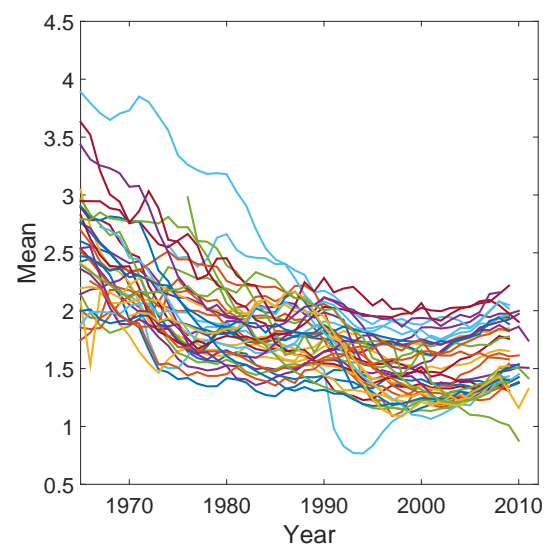

(c)

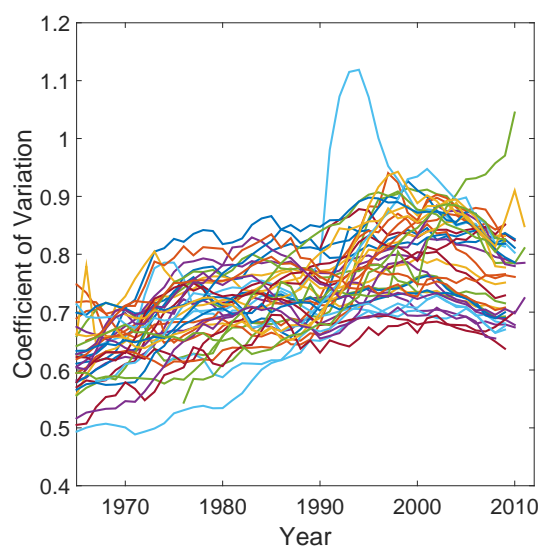

(b) $\quad \mathrm{SD}$ as a function of time

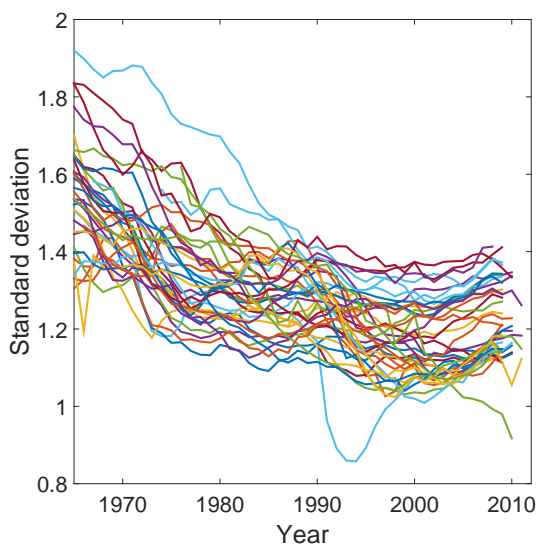

(d) Skewness as a function of time

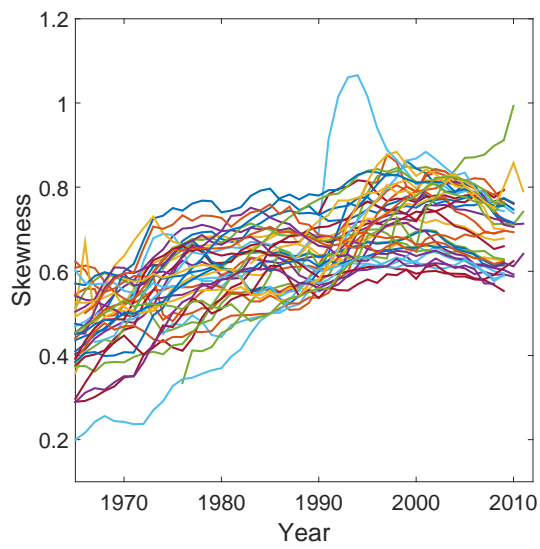

Notes: Mean (a), standard deviation (b), CV (c) and skewness (d) of lifetime reproduction during the period of the second demographic transition for 40 developed countries. The light blue line is East Germany; reasons for its unusual trajectory have been discussed by Witte and Wagner (1995) and Adler (1997).

We analyzed the relationship between the HDI and all four statistics of LRO in 1980 and again in 2009, at which point the HDI had increased notably. Similar to Myrskylä, 
Kohler, and Billari (2009), we find a negative relationship between mean LRO and HDI in the year 1980, but a positive relationship in the year 2009 (see Figure 5(a)). Furthermore, we find a similar reversal in the relationship between HDI and the other statistical properties of LRO (see Figure 5(b-d)). The standard deviation decreased with HDI in the 1980, but increased with HDI in 2009. The CV and skewness show opposite patterns to mean and SD, as both increased with HDI in 1980 and decreased with HDI in 2009. In earlier years, with lower values of HDI, improvements in economic and living conditions led to reduced mean LRO and SD, but increased relative variability as measured by the $\mathrm{CV}$ and increased skewness. In later years, the slopes are reversed (see Table A1 for the regression line equations).

\section{Figure 5: $\quad$ Relationship between statistical properties of LRO and HDI}

(a)

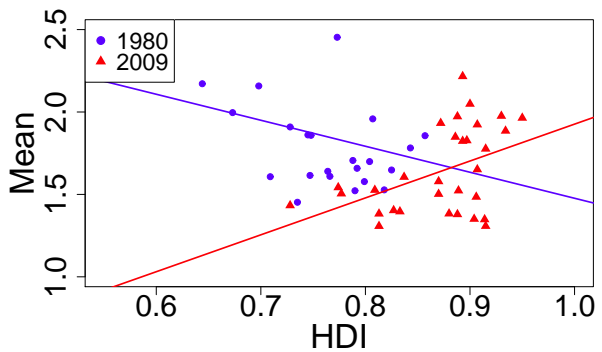

(c)

$\mathrm{CV}$ as a function of HDI

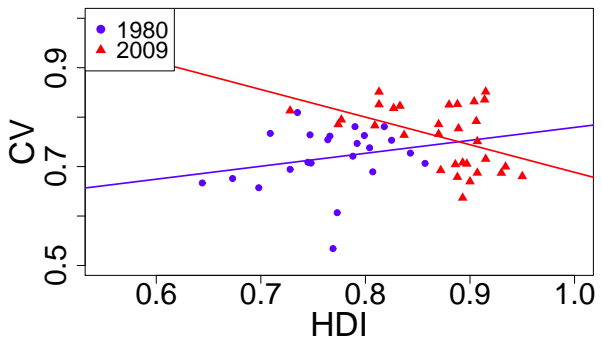

(b) $\quad \mathrm{SD}$ as a function of HDI

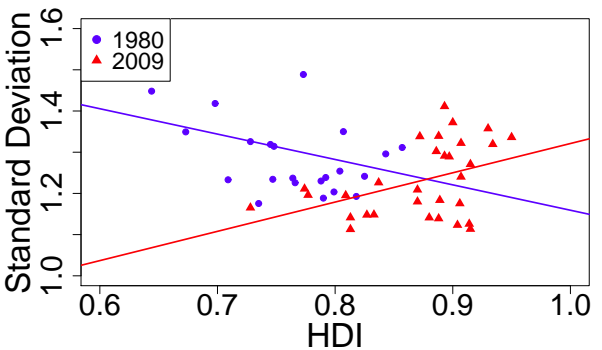

(d) Skewness as a function of HDI

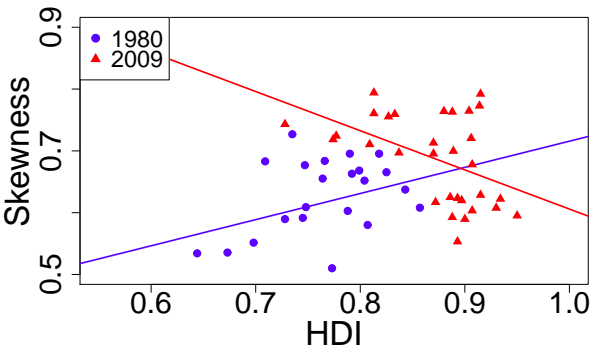

Notes: Relationship of mean, standard deviation, CV and skewness with the Human Development Index (HDI). Blue dots and lines represent this relationship in the year 1980, red triangles and lines represent the relationship in the year 2009. The regression line equations are presented in Table A1 in the Appendix. 
van Daalen \& Caswell: Lifetime reproduction and the second demographic transition

\subsection{Relationships among the statistics of LRO}

The mean, variance, coefficient of variation, and skewness provide a statistical characterization of the LRO implied by the life table and the fertility schedule. When compared across developed countries, a general relationship between these statistics exists. The scatterplot in Figure 6 shows the relationships among all statistics for all countries in the year 2000. The mean and standard deviation of LRO are positively related to each other, as are $\mathrm{CV}$ and skewness. The former statistics are, however, negatively related to the latter (see Table A2 for regression line equations).

When we added data from two additional years (1990 and 2005), the statistics of LRO became slightly less tightly distributed (van Daalen and Caswell, unpublished data). To further explore changes over time, we created phase portraits showing the dynamics of the mean and SD over the historical records available for the countries. Figure 7 shows the time trajectories for 4 countries (Bulgaria, Canada, Japan, and Sweden). The dotted line in the figures is the regression line relating the mean and SD in the scatterplot in Figure 6.

In all four countries, the mean and SD of lifetime reproduction converge to the intercountry regression line. Before the convergence statistics of LRO were more variable both within and between countries. After this convergence countries moved along the line, with both the mean and SD declining at first, before increasing again, as is also shown in Figure 4. The fact that the countries practically "retrace their steps" along the line reinforces the idea of the existence of a universal distribution of LRO to which developed countries appear to converge. Similar patterns were found in all 40 countries we examined.

\section{Discussion}

Analyses based only on means are, by necessity, blind to patterns of variances and other measures of variability. Our analysis, using the Markov chain with rewards model, has documented some such patterns during the second demographic transition. Among a sample of 40 developed countries, repeated patterns were shown to occur over both age and time. In three high fertility populations (the Ache in Paraguay, the Hadza in Tanzania and the Hutterites of North America) mean lifetime reproductive output is, unsurprisingly, higher than in developed countries, but the Ache and Hadza also show a substantial increase in mean remaining LRO between age 0 and age 20 due to high childhood mortality rates. Once individuals have this period of high mortality behind them, mean remaining LRO is higher. The effects of this high childhood mortality on the age patterns of variance and skewness remains to be investigated. 


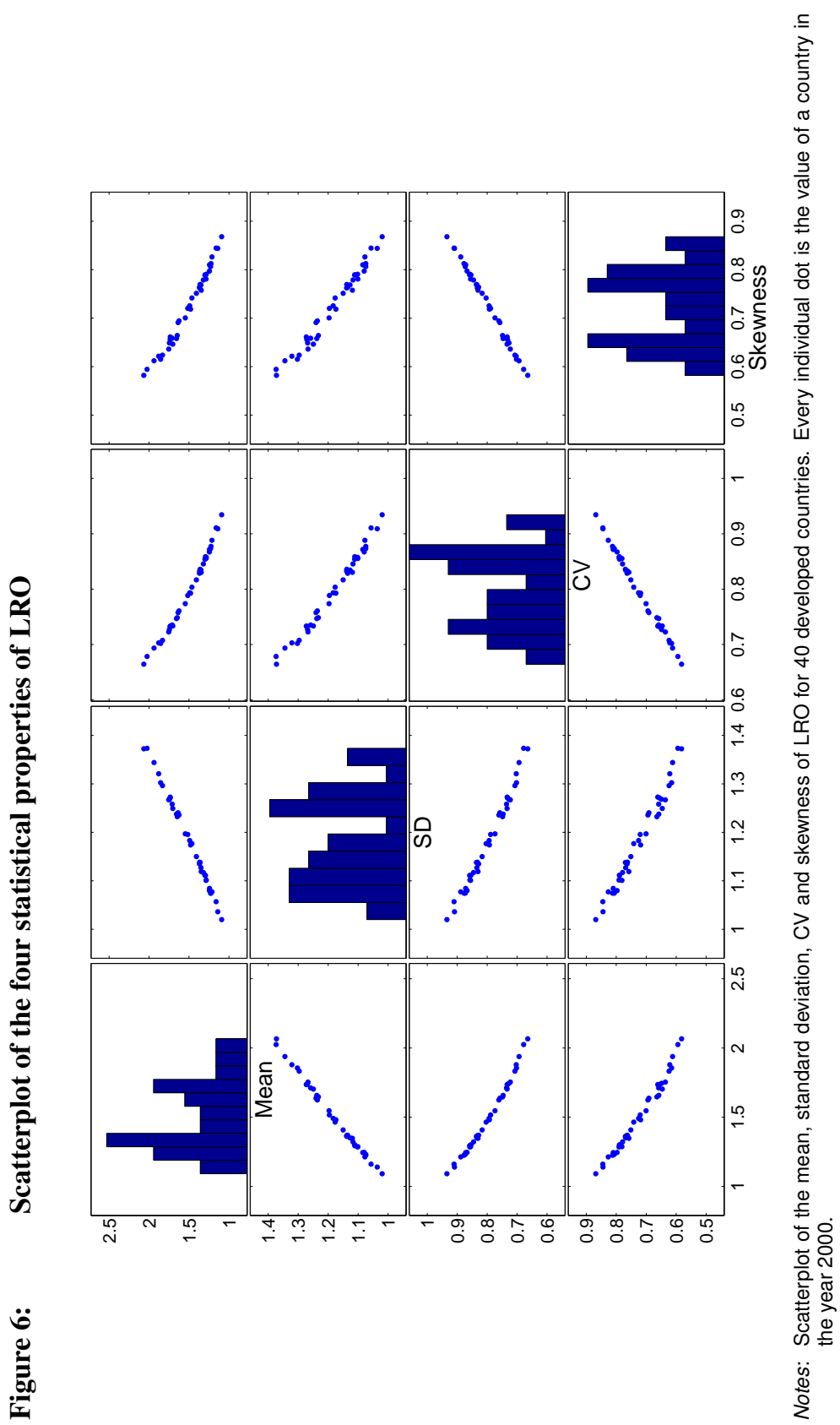


van Daalen \& Caswell: Lifetime reproduction and the second demographic transition

\section{Figure 7: $\quad$ Time trajectories of mean and standard deviation of LRO}

(a)

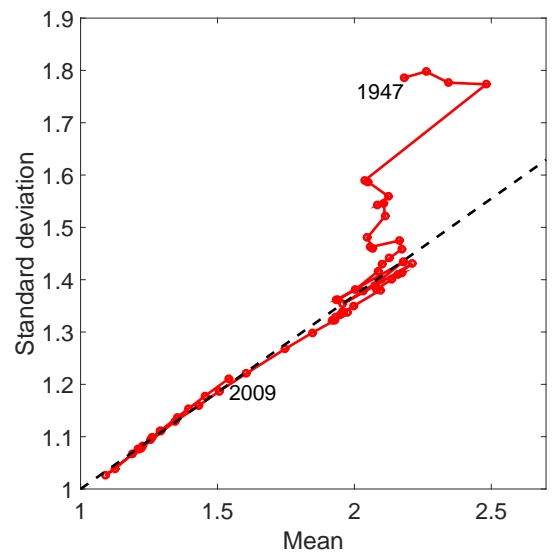

(c)

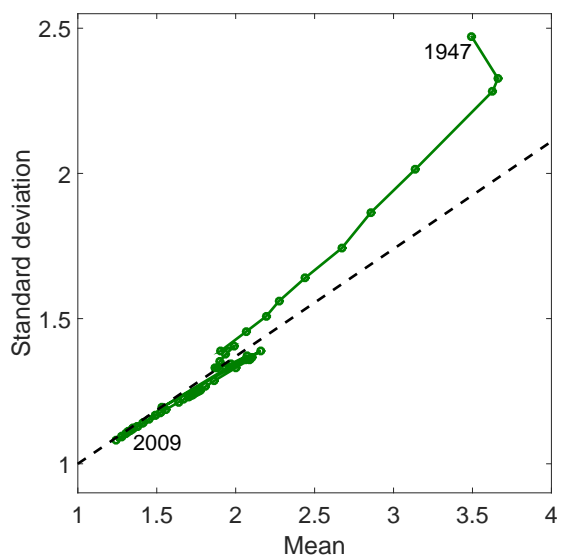

(b) Canada

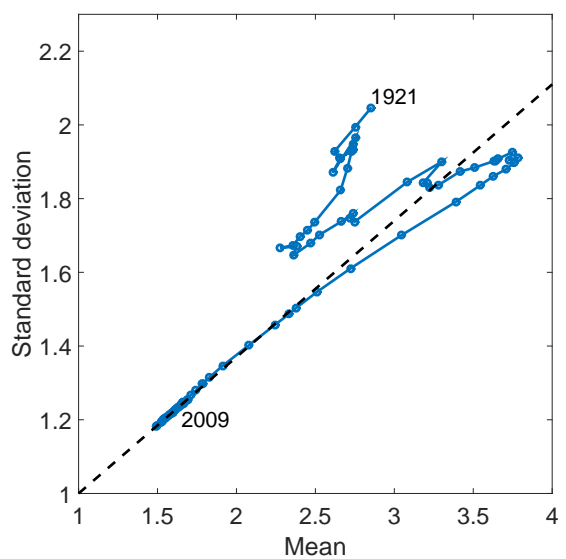

(d) Sweden

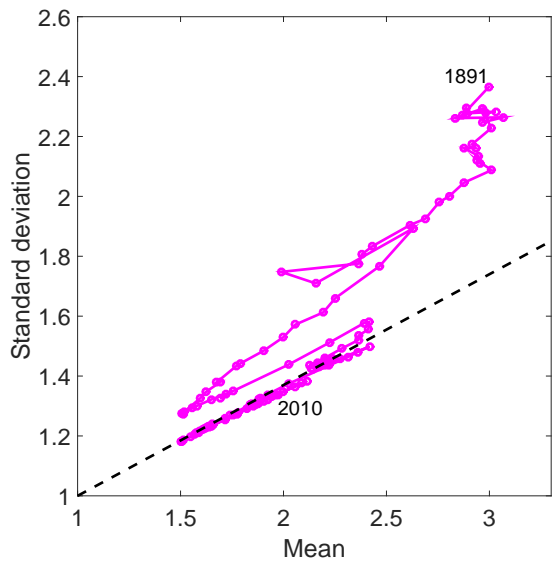

Notes: Trajectories of the mean plotted against standard deviation over time. The starting and ending years are indicated for each trajectory. The dotted lines represent the regression line through the scatterplot of mean and SD shown in Figure 6.

The similarity in patterns among the 40 developed countries suggests a relationship among the statistical properties of lifetime reproduction. Whenever mean LRO changes, 
the other moments change along with it. Therefore, during the second demographic transition, not only mean LRO, but the entire distribution of lifetime reproductive output changed. The transition was characterized by a decreasing mean lifetime reproductive output, a decreasing standard deviation (so a decreasing spread in values), an increasing CV (i.e. an increase in the measure of relative variation) and an increasing, positive skewness (an increase in the degree of asymmetry characterizing the distribution).

The tight link between statistical properties of LRO across different developed countries in the year 2000 (Figure 6) suggests a universal distribution of LRO. If mortality were so low that all individuals survived through their reproductive years, then LRO would be the sum of 50 Bernoulli trials, with a different probability at each age. Such a sum is a random variable with a Poisson-binomial distribution. If the probabilities are small enough, the Poisson-binomial distribution is well approximated by the Poisson distribution (Le Cam 1960; Steele 1994).

The mean and variance of the Poisson distribution are equal, the coefficient of variation is a function of the mean, and the coefficient of variation and skewness are equal. We observe these relationships to some extent in Figure 6 when mortality has become very low in these countries. In earlier years, or in the fixed reward model, the relationships among the statistics of LRO are much looser (van Daalen and Caswell, unpublished data).

\subsection{Individual stochasticity and its components}

Individual variation has received considerable attention in studies of mortality, but very little in studies of fertility. There is a large literature examining patterns of variation in the age at death and a variety of indices of discrepancy in longevity (Anand and Nanthikesan 2000; van Raalte and Caswell 2013). Like all life table calculations, these assume that all individuals experience the same set of specified vital rates, and hence the calculated variation is due to individual stochasticity (van Raalte and Caswell 2013). Studies of fertility have paid much less attention to inter-individual variation of lifetime reproduction. Instead, focus has been largely on expectations of various kinds $\left(R_{0}\right.$ or TFR) and how those expectations change. Our results here are, to our knowledge, the first comparative study of the inter-individual variation in LRO implied by mortality and fertility schedules.

The variance in LRO due to individual stochasticity comes from two sources. Individuals may follow different pathways through life; this variation is generated from the transition matrix $\mathbf{U}$ in equation (1). In the simple age-classified case considered here, the pathway taken by an individual is completely specified by its age at death. ${ }^{6}$ The other component of variance comes from the stochastic nature of reproduction at each age,

\footnotetext{
${ }^{6}$ In more complex or multi-state models, pathways can be more varied; see, e.g., the age $\times$ parity model of Caswell (2014b).
} 
van Daalen \& Caswell: Lifetime reproduction and the second demographic transition

which enters the model through the matrices $\mathbf{R}_{i}$ giving the moments of the reproductive "rewards" in equations (4) and (5).

Mortality rates have declined, and life expectancies increased, during the demographic transition. As a result, the chance that a woman will survive to the end of her reproductive years has increased; hence we expect more and more of the variance in LRO to be accounted for by the stochastic nature of reproduction. To evaluate this, we compare the variance from the full model with the results of a model in which the fertility rewards are fixed, rather than stochastic. In a fixed reward model (Caswell 2011) a fertility of $f_{i}$ implies that every individual of age $i$ produces a fraction $f_{i}$ of a child, rather than producing one or zero children with probabilities $f_{i}$ and $1-f_{i}$.

Figure 8 shows the fraction of the variance in LRO due to the variance in the fertility rewards, as a function of life expectancy, for the developed countries in our dataset. As life expectancy increases, the proportion of variance explained by the randomness in the rewards approaches 1 . We conclude that improvement of health and life expectancy, and the subsequent reduction of the influence of mortality, plays a crucial role in determining the distribution of lifetime reproduction in developed countries.

\subsection{Individual stochasticity}

The inter-individual variation in LRO shown here is a function of individual stochasticity alone. Our results do not incorporate heterogeneity among individuals in mortality or fertility. They could be interpreted as baseline results in comparison to measurements of actual lifetime reproduction (Caswell 2011; Steiner and Tuljapurkar 2012). Adding further dimensions of heterogeneity in addition to age may increase or decrease the variance in LRO (Caswell 2014b). The overall effect of heterogeneity on LRO is an open problem; distinguishing the two effects will require models that incorporate heterogeneity, as frailty models do for studies of mortality.

However, in some cases it is possible to compare the variance due to individual stochasticity, calculated from the demographic rates, to the variance measured among a set of individuals followed over their reproductive lifetimes. This measured variance includes both individual stochasticity and heterogeneity, but such studies are rare, and many of them (e.g., Spuhler 1976) consider only surviving women and/or only married women (Courtiol et al. 2012). In one exceptional study, however, Courtiol et al. (2012) measured the standardized variance in LRO for an entire cohort of Finnish women living in the period 1760-1849, using data from the church books of four Finnish populations. They measured variation by the standardized variance (Crow's $I$; Crow (1958)), which measures the maximum opportunity for selection. Courtiol et al. (2012) found a value close to 2 , which is much higher than the values $(\approx 0.5)$ that we calculate for the countries in our dataset during the transition (Figure 9). Of course, the vital rates in Finland in the 
18th and 19th century are different from those of Europe during the second demographic transition, but this crude comparison suggests that the opportunity for selection is to a large part determined by heterogeneity, with only a relatively small contribution from individual stochasticity. Such comparisons are a valuable tool for understanding observed variance in LRO (Tuljapurkar, Steiner, and Orzack 2009; Steiner and Tuljapurkar 2012; van Daalen and Caswell 2015).

Figure 8: $\quad$ Partitioning the variance in LRO as a function of life expectancy

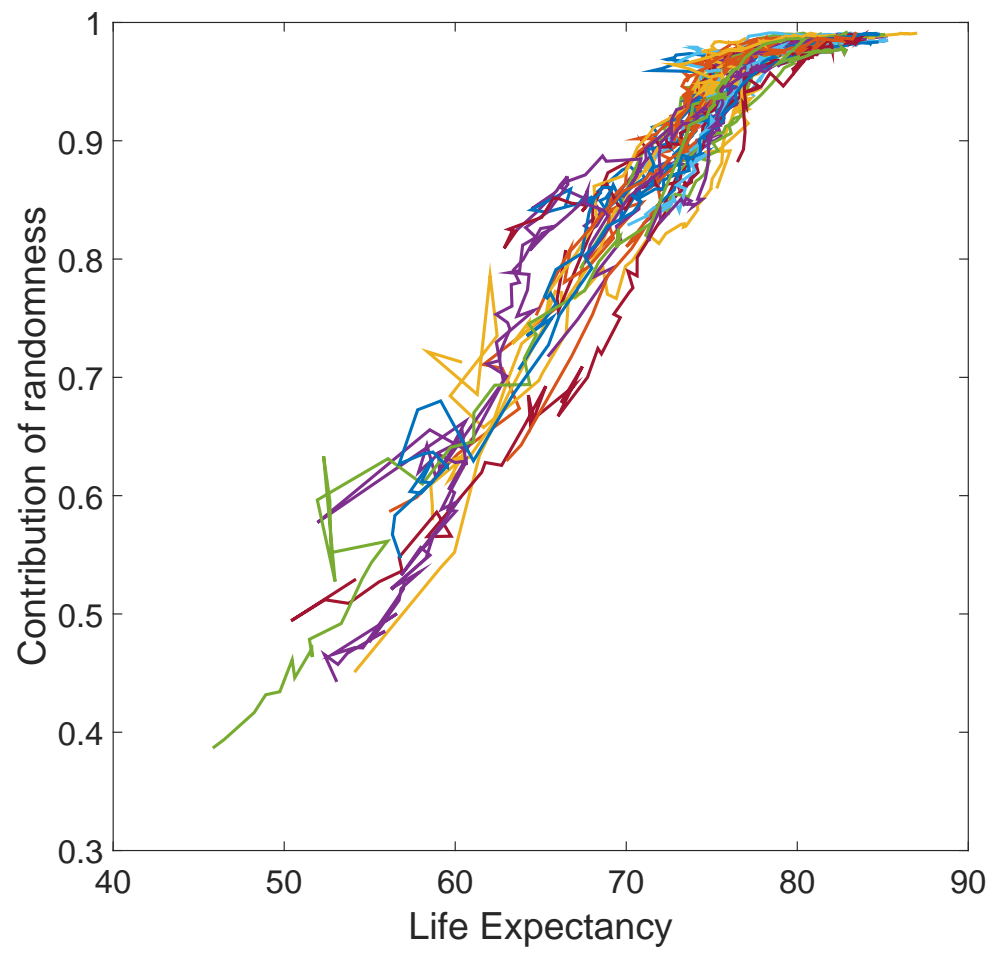

Notes: The proportion of variance in LRO that is due to the randomness in the rewards is shown as a function of life expectancy. Both measures are calculated at every point in time for all 40 developed countries.

Our approach makes it possible to explore the addition of heterogeneity to the model. This can be achieved by developing multistate models that include more details in the reproductive process (Caswell 2014b) or by linking the results to Markov chain models incorporating heterogeneous frailty (Caswell 2014a). Sensitivity analysis of these models 
van Daalen \& Caswell: Lifetime reproduction and the second demographic transition

will show how the statistics of LRO respond to changes in the parameters of the mortality and fertility schedules (Caswell and van Daalen 2015). Finally, we note that the method can be applied to rewards other than reproductive output, including health and longevity (Caswell and Zarulli, unpublished data) and lifetime accumulation of economic rewards (Caswell and Kluge 2015).

Figure 9: $\quad$ Standardized variance due to individual stochasticity compared to observed value for Finland 1760-1849

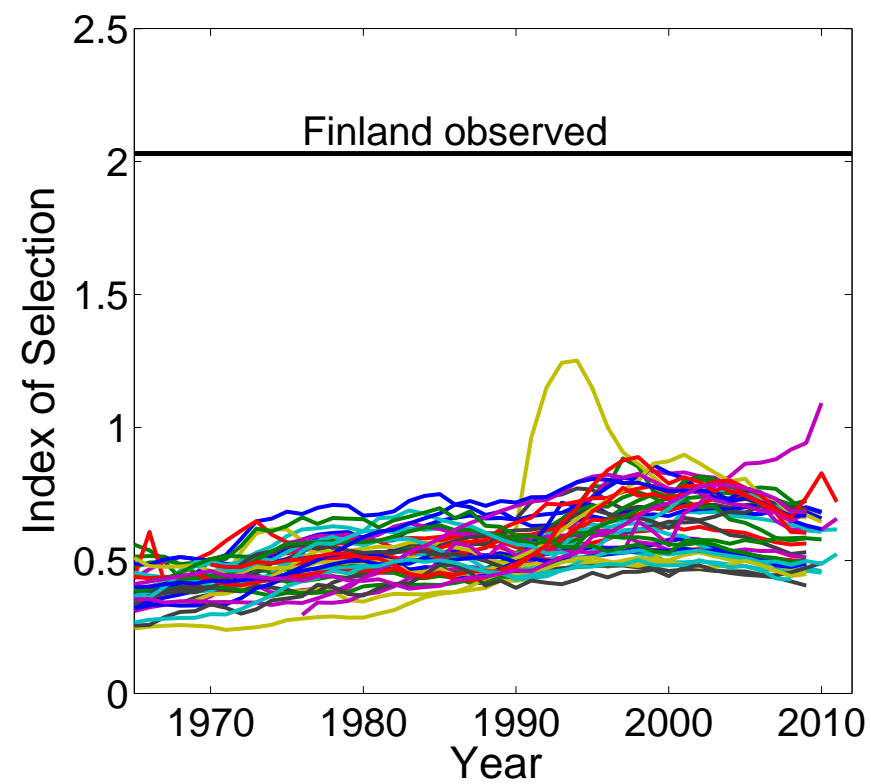

Notes: The standardized variance (Crow's index of selection) for 40 developed countries during the second demographic transition, compared to the standardized variance of LRO among women in Finland living between 1760 and 1849 (Courtiol et al. 2012).

\section{Acknowledgements}

This research was supported by ERC Advanced Grant 322989. We thank Oskar Burger, Josh Goldstein, Mikko Myrskylä, Greg Roth, and Tomáš Sobotka for helpful discussions, and two anonymous reviewers for comments on a previous version. 


\section{References}

Adler, M.A. (1997). Social change and declines in marriage and fertility in eastern Germany. Journal of Marriage and Family 59(1): 37-49. doi:10.2307/353660.

Anand, S. and Nanthikesan, S. (2000). A compilation of length-of-life distribution measures for complete life tables. Working Paper (10) 7, Harvard Center for Population and Development Studies, Harvard School of Public Health.

Blurton Jones, N. (2011). Hadza demography and sociobiology. Retrieved from http://www.sscnet.ucla.edu/anthro/faculty/blurtonjones/hadza-part-1.pdf.

Bongaarts, J. and Sobotka, T. (2012). A demographic explanation for the recent rise in European fertility. Population and Development Review 38(1): 83-120. doi:10.1111/j.1728-4457.2012.00473.x.

Breuer, T., Robbins, A.M., Olejniczak, C., Parnell, R.J., Stokes, E.J., and Robbins, M.M. (2010). Variance in the male reproductive success of western gorillas: Acquiring females is just the beginning. Behavioural Ecology and Sociobiology 64(4): 515-528. doi:10.1007/s00265-009-0867-6.

Brown, D. (1988). Components of lifetime reproductive success. In: Clutton-Brock, T.H. (ed.). Reproductive Success. Chicago: University of Chicago Press: 439-453.

Bryant, J. (2007). Theories of fertility decline and the evidence from development indicators. Population and Development Review 33(1): 101-127. doi:10.1111/j.17284457.2007.00160.x.

Caswell, H. (2001). Matrix Population Models: Construction, Analysis, and Interpretation. Sunderland: Sinauer Associates, 2nd ed.

Caswell, H. (2006). Applications of Markov chains in demography. In: MAM2006: Markov Anniversary Meeting. Raleigh, North Carolina: Boson Books, 319-334.

Caswell, H. (2009). Stage, age and individual stochasticity in demography. Oikos 118: 1763-1782. doi:10.1111/j.1600-0706.2009.17620.x.

Caswell, H. (2011). Beyond $R_{0}$ : Demographic models for variability of lifetime reproductive output. PLoS ONE 6(6): e20809. doi:10.1371/journal.pone.0020809.

Caswell, H. (2014a). A matrix approach to the statistics of longevity in heterogeneous frailty models. Demographic Research 31: 553-592. doi:10.4054/DemRes.2014.31.19.

Caswell, H. (2014b). Statistics of inter-individual variation in lifetime fertility: A Markov chain approach. Paper presented at PAA Annual Meeting, 2014. 
van Daalen \& Caswell: Lifetime reproduction and the second demographic transition

Caswell, H. and Kluge, F.A. (2015). Demography and the statistics of lifetime economic transfers under individual stochasticity. Demographic Research 32: 563-588. doi:10.4054/DemRes.2015.32.19.

Caswell, H. and van Daalen, S.F. (2015). Markov chains with random rewards: Equilibria and sensitivity of long-term accumulation. Preprint.

Clutton-Brock, T.H. (1988). Reproductive Success. Chicago: University of Chicago Press.

Courtiol, A., Pettay, J.E., Jokela, M., Rotkirch, A., and Lummaa, V. (2012). Natural and sexual selection in a monogamous historical human population. Proceedings of the National Academy of Sciences 109(21): 8044-8049. doi:10.1073/pnas.1118174109.

Crow, J.F. (1958). Some possibilities for measuring selection intensities in man. Human Biology 30(1): 1-13.

Cushing, J. and Zhou, Y. (1994). The net reproductive value and stability in matrix population models. Natural Resources Modeling 8(4): 297-333.

Eaton, J.W. and Mayer, A.J. (1953). The social biology of very high fertility among the Hutterites: The demography of a unique population. Human Biology 25(3): 206-264.

Feichtinger, G. (1973). Markovian models for some demographic processes. Statistische Hefte 14: 310-334. doi:10.1007/BF02923066.

Goldstein, J.R., Sobotka, T., and Jasilioniene, A. (2009). The end of "lowest-low" fertility? Population and Development Review 35(4): 663-699. doi:10.1111/j.17284457.2009.00304.x.

Grafen, A. (1988). On the uses of data on lifetime reproductive success. In: CluttonBrock, T.H. (ed.). Reproductive Success. Chicago: University of Chicago Press: 454471.

Gurven, M. and Kaplan, H. (2007). Longevity among hunter-gatherers: A crosscultural examination. Population and Development Review 33(2): 321-365. doi:10.1111/j.1728-4457.2007.00171.x.

Heesterbeek, J.A. (2002). A brief history of R0 and a recipe for its calculation. Acta Biotheoretica 50(3): 189-204. doi:10.1023/A:1016599411804.

Hill, K. and Hurtado, A. (1996). Aché Life History: The Ecology and Demography of a Foraging People. Evolutionary Foundations of Human Behavior Series. New York: Aldine de Gruyter.

Hill, K. and Kaplan, H. (1999). Life history traits in humans: Theory and empirical studies. Annual Review of Anthropology 28: 397-430. 
doi:10.1146/annurev.anthro.28.1.397.

Howard, R.A. (1960). Dynamic Programming and Markov Processes. New York: Technology Press and Wiley.

Human Fertility Collection (2014). Max Planck Institute for Demographic Research (Germany) and the Vienna Institute of Demography (Austria). URL www . fertilitydata.org.

Human Fertility Database (2014). Max Planck Institute for Demographic Research (Germany) and the Vienna Institute of Demography (Austria). URL www . humanfertility.org.

Human Mortality Database (2014). University of California, Berkeley (USA), and Max Planck Institute for Demographic Research (Germany). URL www. mortality . org.

Kirk, D. (1996). Demographic transition theory. Population Studies 50(3): 361-387. doi:10.1080/0032472031000149536.

Le Bras, H. (2008). The Nature of Demography. Princeton, NJ: Princeton University Press.

Le Cam, L. (1960). An approximation theorem for the Poisson binomial distribution. Pacific Journal of Mathematics 10(4): 1181-1197. doi:10.2140/pjm.1960.10.1181.

Lee, R. (2003). The demographic transition: Three centuries of fundamental change. Journal of Economic Perspectives 17(4): 167-190. doi:10.1257/089533003772034943.

Liu, J., Rotkirch, A., and Lummaa, V. (2012). Maternal risk of breeding failure remained low throughout the demographic transitions in fertility and age at first reproduction in Finland. PLoS ONE 7(4): e34898. doi:10.1371/journal.pone.0034898.

Lotka, A.J. (1936). The geographic distribution of intrinsic natural increase in the United States, and an examination of the relation between several measures of net reproductivity. Journal of the American Statistical Association 31: 273-294. doi:10.1080/01621459.1936.10503330.

Myrskylä, M., Kohler, H.P., and Billari, F.C. (2009). Advances in development reverse fertility declines. Nature 460(7256): 741-743. doi:10.1038/nature08230.

Newton, I. (1989). Lifetime reproduction in birds. New York: Academic Press.

Partridge, L. (1989). Lifetime reproductive success and life history evolution. In: Newton, I. (ed.). Lifetime reproduction in birds. New York: Academic Press: 421-440.

Rhodes, E.C. (1940). Population mathematics. I. Journal of the Royal Statistical Society 
van Daalen \& Caswell: Lifetime reproduction and the second demographic transition

103: 61-89. doi:10.2307/2980551.

Robbins, A.M., Stoinski, T., Fawcett, K., and Robbins, M.M. (2011). Lifetime reproductive success of female mountain gorillas. American Journal of Physical Anthropology 146(4): 582-593. doi:10.1002/ajpa.21605.

Spuhler, J.N. (1976). The maximum opportunity for natural selection in some human populations. In: Zubrow, E.B.W. (ed.). Demographic Anthropology: Quantitative Approaches. Albuquerque, New Mexico: University of New Mexico Press: 185-226.

Steele, J.M. (1994). Le Cam's inequality and Poisson approximations. American Mathematical Monthly 101(1): 48-54. doi:10.2307/2325124.

Steiner, U.K. and Tuljapurkar, S. (2012). Neutral theory for life histories and individual variability in fitness components. Proceedings of the National Academy of Sciences 109(12): 4684-4689. doi:10.1073/pnas.1018096109.

Tuljapurkar, S., Steiner, U.K., and Orzack, S.H. (2009). Dynamic heterogeneity in life histories. Ecology Letters 12: 93-106. doi:10.1111/j.1461-0248.2008.01262.x.

United Nations Development Programme (2014). Human Development Index (HDI). Retrieved from http://hdr.undp.org/en/content/humandevelopment-index-hdi, 1 April 2014.

van Daalen, S. and Caswell, H. (2015). Lifetime reproductive output in birds and mammals. (in prep.) .

van Raalte, A.A. and Caswell, H. (2013). Perturbation analysis of indices of lifespan variability. Demography 50(5): 1615-1640. doi:10.1007/s13524-013-0223-3.

Vaupel, J.W., Manton, K.G., and Stallard, E. (1979). The impact of heterogeneity in individual frailty on the dynamics of mortality. Demography 16(3): 439-454. doi:10.2307/2061224.

Wilson, C. (2004). Fertility below replacement level. Science 304(5668): 207-209. doi:10.1126/science.304.5668.207c.

Witte, J.C. and Wagner, G.G. (1995). Declining fertility in East Germany after unification: A demographic response to socioeconomic change. Population and Development Review 21(2): 387-397. doi:10.2307/2137500.

Woofter, T. (1949). The relation of the net reproduction rate to other fertility measures. Journal of the American Statistical Association 44: 501-517. doi:10.1080/01621459.1949.10483323. 


\section{Appendix}

In Table A1, equations are shown for the regression of several statistics of LRO as functions of HDI. These lines, for the year 1980 and the year 2009, are drawn in Figure 5 as well. Table A2 shows the regression lines for the relationships among the statistical properties of LRO.

Table A1: $\quad$ Regression lines for the statistics of LRO as functions of HDI

\begin{tabular}{l|c|r}
\hline \multicolumn{1}{c|}{ Statistic } & \multicolumn{1}{c}{$\mathbf{1 9 8 0}$} & $\mathbf{2 0 0 9}$ \\
\hline Mean & $3.054-0.578 \times \mathrm{HDI}$ & $-0.312+2.239 \times \mathrm{HDI}$ \\
Standard Deviation & $1.775-0.616 \times \mathrm{HDI}$ & $0.611+0.710 \times \mathrm{HDI}$ \\
CV & $0.517+0.262 \times \mathrm{HDI}$ & $1.247-0.558 \times \mathrm{HDI}$ \\
Skewness & $0.293+0.423 \times \mathrm{HDI}$ & $1.240-0.634 \times \mathrm{HDI}$ \\
\hline
\end{tabular}

Notes: Regression lines for the years 1980 and 2009, as shown in Figure 5. 
van Daalen \& Caswell: Lifetime reproduction and the second demographic transition

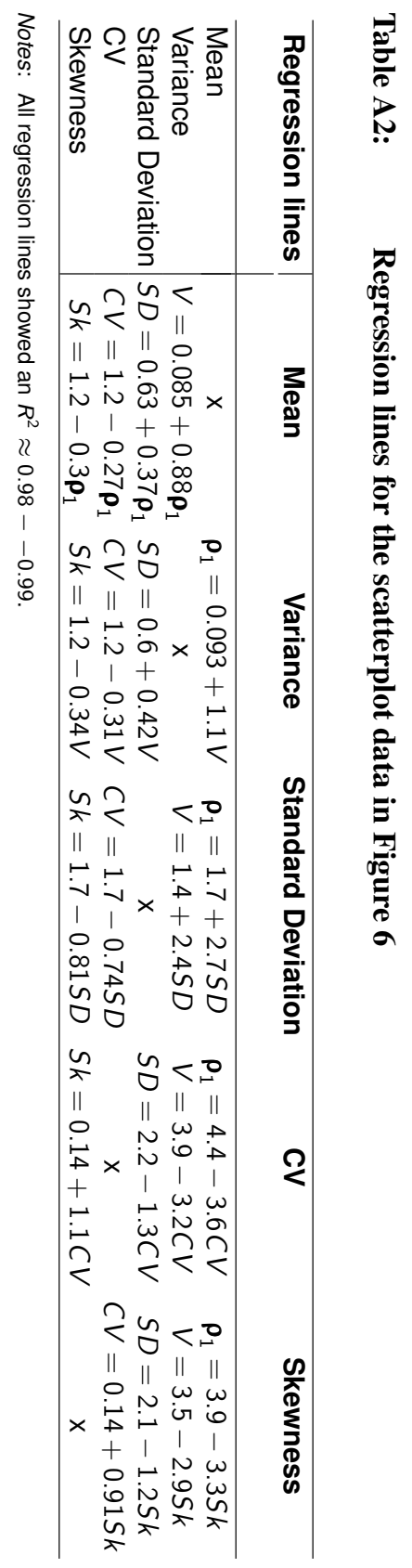

